\title{
Micropulse Transscleral Cyclophotocoagulation
}

\author{
Youssef Abdelmassih ${ }^{1}$, Karim Tomey ${ }^{2}$, Ziad Khoueir ${ }^{3}$ \\ Keywords: Glaucoma therapy, Glaucoma treatment, Micropulse, Micropulse transscleral cyclophotocoagulation. \\ Journal of Current Glaucoma Practice (2021): 10.5005/jp-journals-10078-1298
}

\section{INTRODUCTION}

Treatment of glaucoma aims at lowering intraocular ocular pressure (IOP), either by reducing aqueous humor production or by increasing its outflow through the trabecular or uveoscleral routes, or both. The traditional therapeutic approach consists of non-invasive options in the early phases of the disease (topical and oral medications, and laser therapy such as selective laser trabeculoplasty-SLT), to be followed by invasive surgeries, such as trabeculectomy and glaucoma drainage devices, at a more advanced stage. ${ }^{1}$ Among the classical treatments, continuouswave transscleral cyclophotocoagulation (CW-TSCPC) was left as a last resort when all other medical and surgical treatments were exhausted since it is frequently associated with severe complications such as inflammation, chronic hypotony, and phthisis bulbi. ${ }^{2-5}$ Continuous-wave transscleral cyclophotocoagulation uses an $810 \mathrm{~nm}$ diode laser to achieve IOP lowering via two mechanisms: the destruction of the pigmented secretory ciliary body epithelium, with indirect destruction of the non-pigmented cell layer as well, and increase in the uveoscleral outflow. ${ }^{6,7}$ The past decade has witnessed the introduction of several new technologies to the field of glaucoma, widening the therapeutic options, and increasing the safety profile of the interventions. Minimally invasive glaucoma surgery (MIGS) is becoming more popular and some surgeons are proposing it as an alternative to non-invasive surgeries. ${ }^{8}$ Furthermore, a new TSCPC using micropulse technology (repetitive alteration of on and off laser cycles) was introduced to decrease the side effects related to CW-TSCPC while maintaining a satisfactory efficacy, thus changing its place in the treatment continuum so that it is no longer reserved for end-stage glaucoma. The micropulse transscleral cyclophotocoagulation (mTSCPC) uses a novel MP3 probe and the laser is delivered with the Cyclo G6 Glaucoma Laser System (Iridex Corporation, Mountain View, CA, USA) (Fig. 1). ${ }^{9-13}$ This editorial aims at presenting an overview of the MTSCPC technology.

\section{Treatment Application}

The MP3 probe delivers through its fiberoptic cable an infrared diode laser with a wavelength of $810 \mathrm{~nm}$. The probe is fitted with a plastic contact hemispheric tip through which the fiberoptic cable protrudes $0.7 \mathrm{~mm}$ in depth at $3 \mathrm{~mm}$ from a hemispheric edge, which helps accurate positioning of the laser $3 \mathrm{~mm}$ posterior to the limbus at the level of the pars plana (Figs $2 \mathrm{~A}$ and $\mathrm{B}$ ). The laser delivers a repetitive short-pulse diode laser with adjustable power (frequently around $2,000 \mathrm{~mW} / \mathrm{cm}^{2}$ ) and a duty cycle (proportion of each cycle during which the laser is on) of usually $31.33 \%$ (laser "on" 0.5 ms, laser "off" $1.1 \mathrm{~ms}$ ). The probe is applied using steady pressure, parallel to the visual axis, and moved in a continuous sliding arc along the limbus (painting manner).
${ }^{1}$ Retina Department, Fondation Ophtalmologique Adolphe de Rothschild, Paris, France; Ophthalmology Department, Saint-Joseph University, Faculty of Medicine, Beirut, Lebanon

${ }^{2}$ Glaucoma Department, Beirut Eye and ENT Specialist Hospital, Beirut, Lebanon; Ophthalmology Department, Lebanese American University, Gilbert and Rose-Mary Chagoury School of Medicine, Byblos, Lebanon

${ }^{3}$ Ophthalmology Department, Saint-Joseph University, Faculty of Medicine, Beirut, Lebanon; Glaucoma Department, Beirut Eye and ENT Specialist Hospital, Beirut, Lebanon; Ophthalmology Department, Mayo Clinic, Jacksonville, Florida, USA

Corresponding Author: Ziad Khoueir, Ophthalmology Department, Saint-Joseph University, Faculty of Medicine, Beirut, Lebanon; Glaucoma Department, Beirut Eye and ENT Specialist Hospital, Beirut, Lebanon; Ophthalmology Department, Mayo Clinic, Jacksonville, Florida, USA, Phone: +9611423111, e-mail: ziad@khoueir.com

How to cite this article: Abdelmassih Y, Tomey K, Khoueir Z. Micropulse Transscleral Cyclophotocoagulation. J Curr Glaucoma Pract 2021;15(1):1-7.

Source of support: Nil

Conflict of interest: Dr Khoueir, Consultant Iridex Corporation, Mountain View, CA, USA

The treatment path should avoid the 3 and 9 o'clock meridians, areas of scleral thinning, and areas of previous glaucoma surgery while the treatment is being administered. ${ }^{9-12,14,15}$ Only one study used the stop-and-go motion instead of the painting manner. ${ }^{16}$ Figures 2 and 3 propose tips for performing mTSCPC treatment.

The treatment is usually performed in the operating room under peribulbar or general anesthesia. The surgeon can sometimes use an external lighting source to better localize the position of the ciliary body, especially in the pediatric population, or in eyes with high myopia or buphthalmia. The postoperative treatment consists of topical corticosteroid and possibly non-steroidal antiinflammatory drops, tapered slowly during the postoperative period. Antiglaucoma drops are kept in the postoperative period and then are either stopped, tapered, or kept at the physician's discretion. ${ }^{9}$ A new revised MP3 rev 2 probe released in 2019 offers better viewing of the treated area and easier treatment application, especially in small and sunken eyes. It also offers more intuitive probe orientation and better light coupling (Fig. 4).

\section{Mechanism of Action}

The mechanism of action of the MTSCPC is not well understood and several mechanisms have been suggested. 

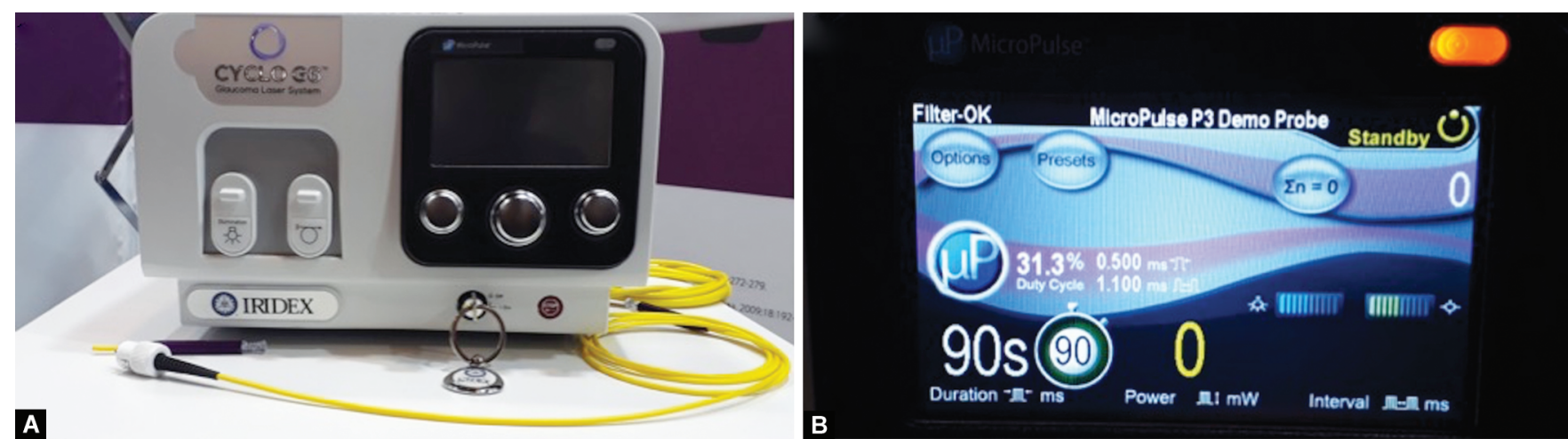

Figs 1 A and B: (A) Cyclo G6 Glaucoma Laser System machine (Iridex Corporation, Mountain View, CA, USA); (B) Welcome screen of the machine with a standard $31.3 \%$ duty cycle and 90 seconds treatment duration
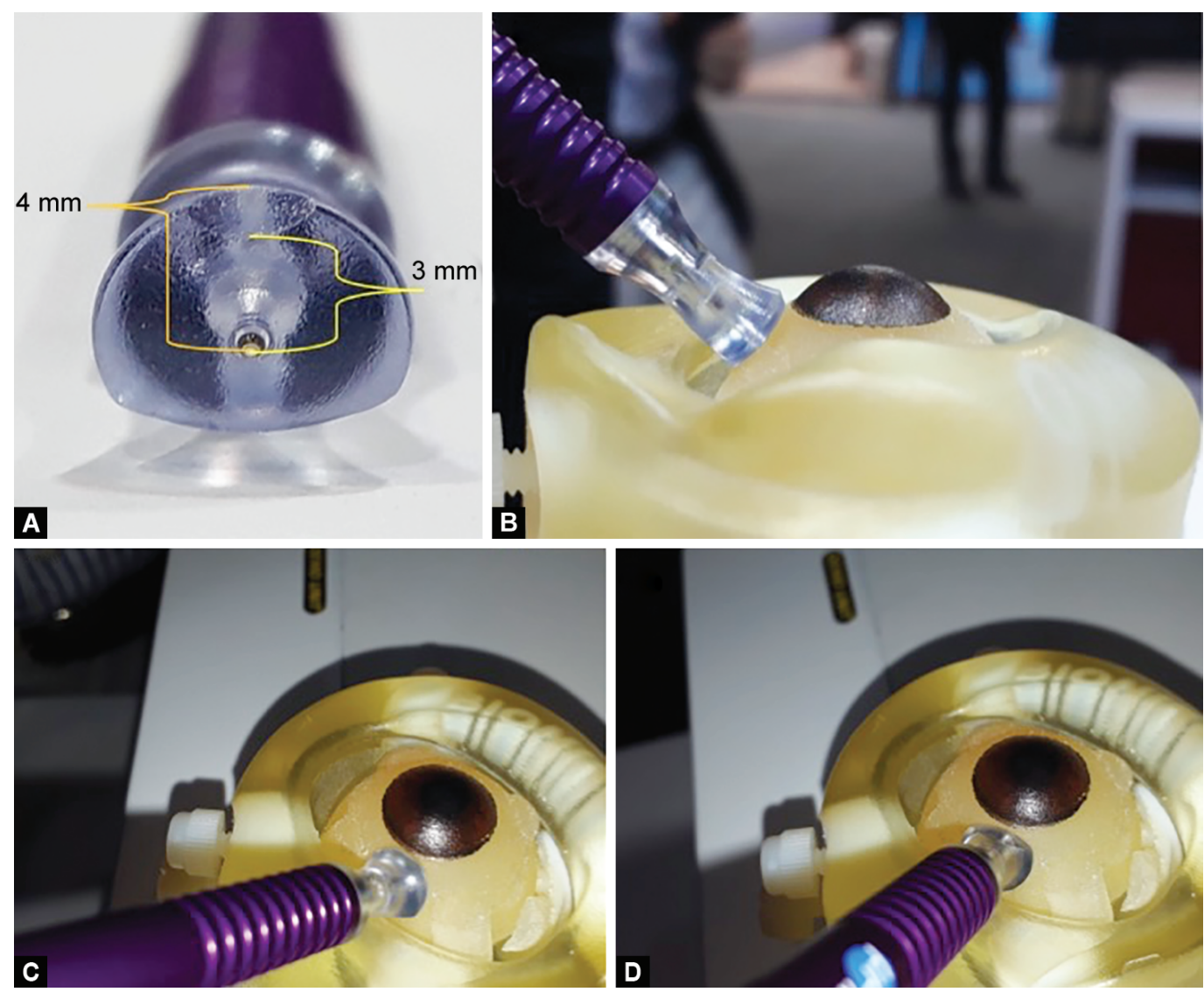

Figs 2A to D: (A) MP3 probe showing 3 and $4 \mathrm{~mm}$ distance from the fiber tip to the probe edge to help deliver the treatment at the right distance from the limbus; (B) The probe should be perpendicular to the globe, not too flat, and not too steep; (C) The anterior curved edge of the probe should be positioned at the limbus or $0.5 \mathrm{~mm}$ behind the limbus; (D) The probe should not be in this position; the flat side of the probe should be towards the lid

\section{Decreased Aqueous Humor Production}

The $810 \mathrm{~nm}$-wavelength diode laser is absorbed mainly by the pigmented epithelium of the ciliary body. It is believed that the "on" cycle helps achieves the coagulation threshold of the pigmented ciliary epithelium, while the "off" cycle limits the accumulation of heat and permits thermal dissipation in the adjacent cells, resulting in less disruption of the non-pigmented epithelium and the ciliary stroma. ${ }^{17}$ This limited rise of temperature secondary to laser application denatures proteins faster than the natural biological cell repair mechanism, resulting in a reduction of aqueous humor production without any macroscopic tissue alterations. Anterior segment OCT and ultrasound biomicroscopy have shown no major changes in the ciliary body or anterior segment anatomy. ${ }^{18,19}$ Moussa et al. found that MTSCPC did not cause significant damage to the ciliary body (epithelium destruction, separation of the pigmented ciliary epithelium from the stroma and stromal coagulation). These findings suggest that the IOP-lowering mechanism of MTSCPC may be independent of aqueous production. ${ }^{19}$ 

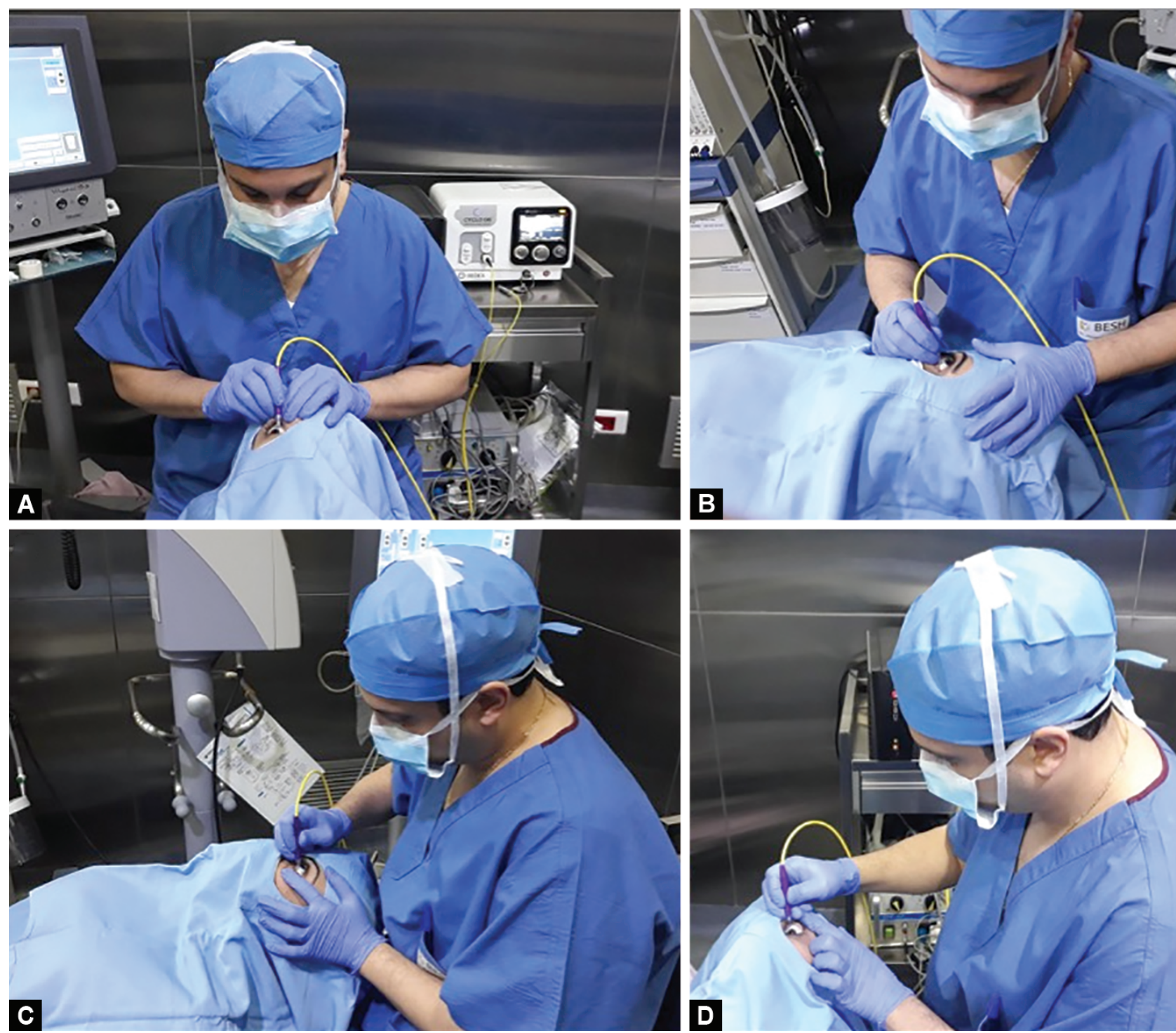

Figs 3A to D: (A and B) Preferred right-handed surgeon's disposition while treating a right eye one hemisphere at a time; (C and D) Preferred righthanded surgeon's disposition while treating a left eye

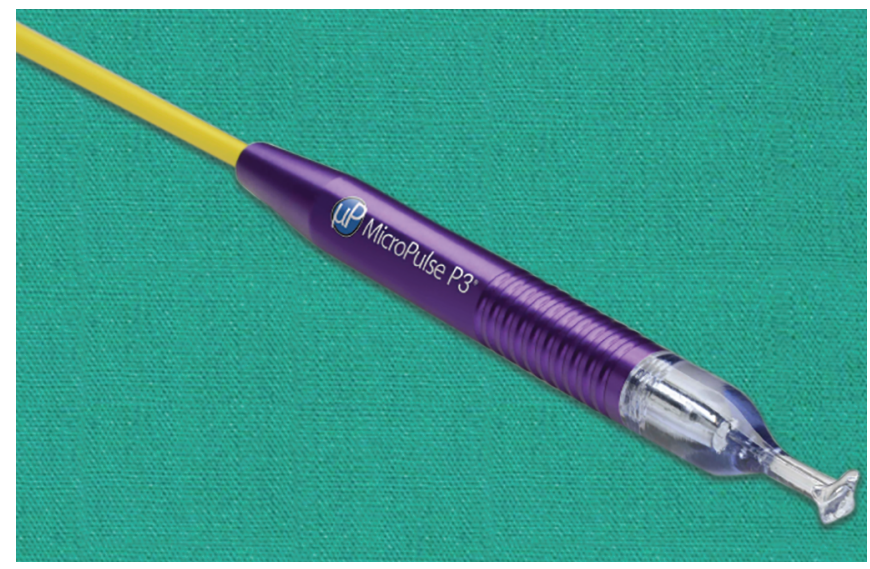

Fig. 4: MP3 rev2 probe gives better viewing of the treated area, a more intuitive orientation and an easier treatment application with better light coupling

\section{Increased Uveoscleral Outflow}

There is no direct evidence on the effect of MTSCPC on uveoscleral outflow. However, this effect was directly observed with CW-TSCP, both histologically ${ }^{20}$ and with the help of tracer-particle perfusion. ${ }^{21}$ Also, an increase in uveoscleral outflow has been suggested indirectly through the observation of an increase in choroidal thickness observed in patients treated successfully with mTSCPC, even though the sample was too small to show a statistically significant difference. $^{22}$

\section{Increased Trabecular Meshwork Outflow}

A third and final mechanism is the action of the laser on the longitudinal fibers of the ciliary body, which leads to its contraction and causes a displacement of the scleral spur, thus changing the configuration of the trabecular meshwork and increasing the outflow through this pathway. This effect is similar to the mechanism of pilocarpine in lowering IOP. It has been demonstrated that this effect is energy-dependent, meaning 
that the higher the energy applied the lower the recovery of the contracted ciliary muscle, resulting in a more sustained pilocarpine-like effect. ${ }^{23,24}$

\section{mTSCPC vs CW-TSCPC}

Only a few studies have compared the safety and efficacy of MTSCPC to CW-TSPC and demonstrated a better safety profile of mTSCPC with equal efficacy. ${ }^{10,25}$ In a prospective randomized study including patients with refractory advanced glaucoma, Aquino et al. reported a comparable efficacy between MTSCPC and CW-TSCPC, with a respective successful outcome defined as an IOP between 6 and 21 $\mathrm{mm} \mathrm{Hg}$ and at least $30 \%$ reduction with or without medication at 18 months in 52 and $30 \%$ of included patients. Furthermore, mTSCPC was significantly safer than CW-TSCPC, with only one patient developing a decrease in his visual acuity, one patient prolonged inflammation, and one patient scleral thinning. ${ }^{10}$

Similar results were observed in the pediatric population with refractory glaucoma. There was a tendency toward lower IOP at 2 weeks and 3 months and a higher success rate, defined as an IOP of $5-21 \mathrm{~mm} \mathrm{Hg}$, at 6 months, without reaching statistical significance $(71 \%$ in the mTSCPC group vs $46 \%$ in the CW-TSCPC; $p=0.1)$. No children developed vision-threatening complications in the MTSCPC group compared with 3 children in the CW-TSCPC group (one eye developed phthisis bulbi and two eyes severe pain and inflammation). ${ }^{25}$

\section{Indications}

The first studies reporting on the outcomes of MTSCPC in glaucoma included only cases with advanced uncontrolled glaucoma. ${ }^{10,11,26-28}$ More recent ones included a wide variety of glaucoma stages, ranging from mild cases with a good central vision to more advanced cases. ${ }^{22,29-32}$ Micropulse transscleral cyclophotocoagulation has been proposed for both pediatric and adult populations, and all glaucoma types as well as for patients with penetrating keratoplasty. 9,14,25,30,33,34 Primary open-angle glaucoma was the most frequently included etiology. $9,13,35$

Other glaucoma etiologies in the adult population were: angle-closure, pigmentary, silicone-oil-induced, malignant, pseudoexfoliation, neovascular, post-traumatic, and post-corneal transplant. ${ }^{9,14,25,30,33}$

Glaucoma etiologies in the pediatric population included: congenital, aniridia, Peter's anomaly, microspherophakia, Sturge-Weber, aphakia, and persistent hyperplastic primary vitreous. $^{14,25}$

\section{Parameters of the Laser}

Several parameters have been tried to find the most adequate treatment duration to achieve the most effective results with the least complication rates. Up to this date, the ideal parameters have not been established yet. Four variables affect the energy delivered to the tissues:

- Treatment duration, which is the main parameter that varies from one physician to another. It represents the total duration of treatment application and ranges between 100 and 360 seconds.

- Sweeping speed (dwell time), which can be fast around 10 seconds per excursion or slow up to 60 seconds per excursion.
- Power, which is usually set to $2,000 \mathrm{~mW}$, but a few studies have used a power ranging between 2,000 and $2,500 \mathrm{~mW} / \mathrm{cm}^{2} .29,35,36$

- Duty cycle preset to 25-31.3\%. Keilani et al. compared $25 \%$ duty cycle to $31.3 \%$ and found that the latter was more effective but induced more inflammation. ${ }^{37}$

To facilitate comparison between studies using different parameters, Johnstone et al. proposed a formula to calculate the delivered energy in Joules $(J)$. Joules $(J)=$ power in Watts $(W) \times$ total treatment duration in seconds $(s) \times$ ON cycle (31.3\% for example). ${ }^{24}$ Furthermore, the IOP-lowering effect is positively correlated with the duration of treatment application, and therefore the total energy. However, care must be taken when using the upper levels of total energy, as it seems to be associated with higher complication rates.

Total energy applied in studies ranged between $62 \mathrm{~J}$ (total application time of 100 seconds) and $225 \mathrm{~J}$ (total application time of 360 seconds). The energy levels applied can be categorized into low, intermediate, and high:

- Low energy levels (62-100J; 100-160 seconds of treatment at $2,000 \mathrm{~mW}){ }^{10,11,35,36,38-40}$

Low energy levels had the lowest complication rates but also a more limited efficacy. Sanchez et al. applied energy levels ranging between $62 \mathrm{~mW}$ and $112 \mathrm{~mW}$ and found an overall success rate of $27.3 \%: 75 \%$ in the $112 \mathrm{~J}$ group, $21.4 \%$ in the $100 \mathrm{~J}$ group, while all patients in the $62 \mathrm{~J}$ group failed. ${ }^{40}$ Souissi et al. reported a similarly low success rate of $35 \%$ with an energy level of $100 \mathrm{~J} .{ }^{26}$ However, Aquino et al. reported a success rate of $75 \%$ at 12 months and $52 \%$ at 18 months using $100 \mathrm{~J}$ energy with almost half of the patients requiring at least two treatment sessions. ${ }^{10}$ Sarrafpour et al. proposed a different treatment approach by using fixed treatment duration and adapting the power of the laser to the visual acuity of patients (light perception or worse: $2,500 \mathrm{~mW}$, hand motion or count fingers: 2,400 mW, 20/400-20/80: 2,250 mW, and 20/70-20/20: $2,000 \mathrm{~mW}$ ) and reported that IOP control depended on the power used. ${ }^{35}$

- Intermediate energy levels (112-200J; 180 to 240 seconds of treatment at 2,000 mW) $)^{9,22,28,29,33,34}$

Studies using intermediate energy between 112 and $150 \mathrm{~J}$ had the best safety/efficacy profile. Zaarour et al. reported on the outcomes in 75 eyes of 69 patients with advanced refractory glaucoma using the fixed parameters of 180 seconds with 2000 $\mathrm{mW}$ of power and a duty cycle of $31.3 \%$. The success rate at one year was $73.3 \%$ with a $35.4 \%$ decrease of IOP from baseline. No serious complications were reported. ${ }^{9}$ Yelenskiy, et al. reported similar results using the following laser parameters: $2,000 \mathrm{~mW}$, $180-240$ seconds, and $31.3 \%$ duty cycle. Only four cases (2\%) of transient cystoid macular edema were reported. ${ }^{33}$

- High energy levels (around 200 to 225J; 320 to 360 seconds of treatment at 2,000 $\mathrm{mW})^{12,14,16,30,31}$

Emanuel et al. used a mean treatment duration of 319 seconds (range: 180-360 seconds) and found a success rate of $75 \%$ at 1 year, with an average of $41.2 \%$ of IOP decrease. However, complications were reported in around $45 \%$ of cases, with persistent inflammation and loss of visual acuity being the most common. ${ }^{12}$ Similarly, Williams et al. reported a success rate 
of $67 \%$ and an average of $51 \%$ decrease in IOP, but with $26 \%$ of patients developing prolonged inflammation and $17 \%$ a loss of visual acuity at 3 months. ${ }^{16}$ However, double-session mTSCPC that delivers laser treatment for 320 seconds but alternating 80 -second sessions between the upper and lower quadrants was found to have similar success rates with lower complication rates. The difference could be due to better dissipation of heat between sessions or to different glaucoma types between studies. ${ }^{30,31}$

Due to the large disparities in treatment parameters, there is no consensus as to the optimal treatment duration and power. In an attempt to tackle this question, Sanchez et al. suggested, based on the experimental study of Johnstone et al. and published literature, that the delivered energy with the best safety/ efficacy profile was between 112 and $150 \mathrm{~J}$. In fact, at $150 \mathrm{~J}$ the contraction of the ciliary muscle becomes permanent with no more relaxation or recoil of the muscle. Contraction of the ciliary body (as mentioned above in the mechanism of action section) helps change the configuration of the trabecular meshwork, thus increasing the aqueous outflow. ${ }^{23,24}$

\section{mTSCPC in the Pediatric Age-groups ${ }^{14,25}$}

Few studies have reported on the safety of MTSCPC in the pediatric population but with varying results as far as success.

Abdelrahman and El Sayed compared the outcomes of mTSCPC to those of CW-TSCPC in refractory pediatric glaucoma and found a success rate of $71 \%$ at the last follow-up in the MTSCPC group (17 eyes), with a mean IOP reduction of $63 \% .^{25}$

Lee et al., on the other hand, reported a short-lived efficacy of MTSCPC in children, with a success rate of $22 \%$ and a mean decrease of IOP of $21 \%$, compared with 72 and $33 \%$, respectively, in the adult population. ${ }^{14}$

The discrepancy in the results could be due to the variability of ciliary body position, the wide variety of glaucoma etiologies in the pediatric population, the duration of follow-up, the extent of treatment (2 vs 4 quadrants) and the possibility of retreatment.

\section{Complications}

Micropulse transscleral cyclophotocoagulation seems to have a better safety profile than CW-TSCPC, with less sight-threatening complications. Complication rates have varied greatly between studies and depended mostly on the energy levels used, being relatively minimal with low levels and reaching $45 \%$ with the high levels. ${ }^{12,32}$ The most frequent complications were persistent anterior chamber inflammation ( $>3$ months), loss of best-corrected visual acuity, hypotony (early, late and persistent), and cystoid macular edema. In phakic patients, especially those with good central vision, cataract progression was the most frequent complication and resulted in a decrease in visual acuity. It was found in $40 \%$ of phakic patients in the study of Varikuti et al. ${ }^{32}$ However, it is still unclear if cataract formation was the result of natural progression or accelerated by the inflammation after mTSCPC.

Less frequent complications included: corneal edema, IOP spikes, severe inflammation with fibrin, transient hyphema, mydriasis, scleral thinning, and serous choroidal detachment that spontaneously resolved. , $12,16,29,32,41^{\text {Despite being a safe }}$ procedure, vision-threatening complications. Williams et al. reported two cases of phthisis bulbi after using high energy in patients with advanced glaucoma. ${ }^{16}$ Perez et al. reported neurotrophic keratitis in two diabetic patients who were predisposed to corneal sensation, following 160 seconds of laser treatment. ${ }^{42}$ Similarly, Kim et al. reported a case of neurotrophic keratitis after 180 seconds of treatment in an 81-year-old patient with no history of diabetes; but this resolved after 3 months of treatment, leaving a central corneal scar. ${ }^{43}$ Prager and Anchala reported a case of suprachoroidal hemorrhage in the setting of postprocedural (180 seconds) hypotony, in a patient with multiple medical comorbidities including coronary artery disease on anticoagulation, hypertension, and diabetes. ${ }^{44}$ Kiyama et al. reported a case of conjunctival laceration that needed suturing, following $\mathrm{MTSCPC}$ treatment in a patient that developed subconjunctival hemorrhage after subTenon injection. ${ }^{45}$ Chan et al. reported a case of acute subepithelial hydrops that resulted in an epithelial defect, but resolved without corneal scarring, in a patient who received $\mathrm{MTSCPC}$ eight days after phacoemulsification. ${ }^{46}$

\section{Conclusion}

Micropulse transscleral cyclophotocoagulation is a safe and effective modality of treatment for glaucoma. It can be used in mild to advanced glaucoma in both children and adults. Several treatment parameters and protocols have been proposed, but still no consensus has been reached yet. Moderate power seems to have the best safety and efficacy profile. Despite its safety profile, mTSCPC has been associated with vision-threatening complications, hence care must be taken when selecting the patients and choosing the treatment parameters.

\section{References}

1. Weinreb RN, Aung T, Medeiros FA. The pathophysiology and treatment of glaucoma: a review. JAMA 2014;311(18):1901-1911. DOI: 10.1001/jama.2014.3192.

2. Pastor SA, Singh K, Lee DA, et al. Cyclophotocoagulation: a report by the American Academy of Ophthalmology. Ophthalmology 2001;108(11):2130-2138. DOI: 10.1016/s0161-6420(01) 00889-2.

3. Ramli N, Htoon $\mathrm{HM}, \mathrm{Ho} \mathrm{CL}$, et al. Risk factors for hypotony after transscleral diode cyclophotocoagulation. J Glaucoma 2012;21(3):169-173. DOI: 10.1097/IJG.0b013e318207091a.

4. Osman EA, Al-Muammar A, Mousa A, et al. Controlled cyclophotocoagulation with diode laser in refractory glaucoma and long term follow up at King Abdulaziz University Hospital, Riyadh. Saudi J Ophthalmol Off J Saudi Ophthalmol Soc 2010;24(1):9-13. DOI: 10.1016/j.sjopt.2009.12.002.

5. Ansari E, Gandhewar J.Long-term efficacy and visual acuity following transscleral diode laser photocoagulation in cases of refractory and non-refractory glaucoma. Eye Lond Engl 2007;21(7):936-940. DOI: 10.1038/sj.eye.6702345.

6. Frezzotti P, Mittica V, Martone G, et al. Longterm follow-up of diode laser transscleral cyclophotocoagulation in the treatment of refractory glaucoma. Acta Ophthalmol (Copenh) 2010;88(1):150-155. DOI: 10.1111/j.1755-3768.2008.01354.x.

7. Grueb M, Rohrbach JM, Bartz-Schmidt KU, et al. Transscleral diode laser cyclophotocoagulation as primary and secondary surgical treatment in primary open-angle and pseudoexfoliatve glaucoma. Long-term clinical outcomes. Graefes Arch Clin Exp Ophthalmol Albrecht Von Graefes Arch Klin Exp Ophthalmol 2006;244(10):12931299. DOI: $10.1007 / \mathrm{s} 00417-006-0280-z$

8. Lavia C, Dallorto L, Maule M, et al. Minimally-invasive glaucoma surgeries (MIGS) for open angle glaucoma: a systematic review and 
meta-analysis. PLoS ONE 2017;12(8):e0183142. DOI: 10.1371/journal. pone.0183142.

9. Zaarour K, Abdelmassih Y, Arej N, et al. Outcomes of micropulse transscleral cyclophotocoagulation in uncontrolled glaucoma patients. J Glaucoma 2019;28(3):270-275. DOI: 10.1097/ IJG.0000000000001174.

10. Aquino MCD, Barton K, Tan AMWT, et al. Micropulse versus continuous wave transscleral diode cyclophotocoagulation in refractory glaucoma: a randomized exploratory study. Clin Experiment Ophthalmol 2015;43(1):40-46. DOI: 10.1111/ceo.12360.

11. Tan AM, Chockalingam M, Aquino MC, et al. Micropulse transscleral diode laser cyclophotocoagulation in the treatment of refractory glaucoma. Clin Experiment Ophthalmol 2010;38(3):266-272. DOI: 10.1111/j.1442-9071.2010.02238.x.

12. Emanuel ME, Grover DS, Fellman RL, et al. Micropulse cyclophotocoagulation: initial results in refractory glaucoma. J Glaucoma 2017;26(8):726-729. DOI: 10.1097/IJG.00000000 00000715.

13. Sanchez FG, Peirano-Bonomi JC, Brossard Barbosa N, et al. Update on micropulse transscleral cyclophotocoagulation. J Glaucoma 2020;29(7):598-603. DOI: 10.1097/IJG.0000000000001539.

14. Lee JH, Shi Y, Amoozgar B, et al. Outcome of Micropulse laser transscleral cyclophotocoagulation on pediatric versus adult glaucoma patients. J Glaucoma 2017;26(10):936-939. DOI: 10.1097/ IJG.0000000000000757.

15. Kuchar S, Moster MR, Reamer CB, et al. Treatment outcomes of micropulse transscleral cyclophotocoagulation in advanced glaucoma. Lasers Med Sci 2016;31(2):393-396. DOI: 10.1007/s10103015-1856-9.

16. Williams AL, Moster MR, Rahmatnejad K, et al. Clinical efficacy and safety profile of Micropulse transscleral cyclophotocoagulation in refractory glaucoma. J Glaucoma 2018;27(5):445-449. DOI: 10.1097/ IJG.0000000000000934.

17. Dastiridou Al, Katsanos A, Denis P, et al. Cyclodestructive procedures in glaucoma: a review of current and emerging options. Adv Ther 2018;35(12):2103-2127. DOI: 10.1007/s12325-018-0837-3.

18. Amoozgar B, Phan EN, Lin SC, et al. Update on ciliary body laser procedures. Curr Opin Ophthalmol 2017;28(2):181-186. DOI: 10.1097/ ICU.0000000000000351.

19. Moussa K, Feinstein M, Pekmezci M, et al. Histologic changes following continuous wave and micropulse transscleral cyclophotocoagulation: a randomized comparative study. TransI Vis Sci Technol 2020;9(5):2222. DOI: 10.1167/tvst.9.5.22.

20. Schubert HD, Agarwala A. Quantitative CW Nd:YAG pars plana transscleral photocoagulation in postmortem eyes. Ophthalmic Surg 1990;21(12):835-839.

21. Liu GJ, Mizukawa A, Okisaka S. Mechanism of intraocular pressure decrease after contact transscleral continuous-wave Nd:YAG laser cyclophotocoagulation. Ophthalmic Res 1994;26(2):65-79. DOI: 10.1159/000267395.

22. Barac R, Vuzitas M, Balta F. Choroidal thickness increase after micropulse transscleral cyclophotocoagulation. Romanian J Ophthalmol 2018;62(2):144-148. DOI: 10.22336/rjo.2018.21.

23. Sanchez FG, Peirano-Bonomi JC, Grippo TM. Micropulse transscleral cyclophotocoagulation: a hypothesis for the ideal parameters. Med Hypothesis Discov Innov Ophthalmol J 2018;7(3):94-100.

24. Johnstone MA, Song S, Padilla S, et al. Microscope real-time video (MRTV), high-resolution OCT (HR-OCT) \& histopathology (HP) to assess how transcleral micropulse laser (TML) affects the sclera, ciliary body (CB), muscle (CM), secretory epithelium (CBSE), suprachoroidal space (SCS) \& aqueous outflow system. Invest Ophthalmol Vis Sci 2019;60(9):2825-2825.

25. Abdelrahman AM, El Sayed YM. Micropulse versus continuous wave transscleral cyclophotocoagulation in refractory pediatric glaucoma. J Glaucoma 2018 ;27(10):900-905. DOI: 10.1097/ IJG.0000000000001053.
26. Souissi S, Baudouin C, Labbé A, et al. Micropulse transscleral cyclophotocoagulation using a standard protocol in patients with refractory glaucoma naive of cyclodestruction. Eur J Ophthalmol 2019;31(1):112-119. DOI: 10.1177/1120672119877586.

27. Garcia GA, Nguyen CV, Yelenskiy A, et al. Micropulse transscleral diode laser cyclophotocoagulation in refractory glaucoma: short-term efficacy, safety, and impact of surgical history on outcomes. Ophthalmol Glaucoma 2019;2(6):402-412. DOI: 10.1016/j. ogla.2019.08.009.

28. Preda MA, Karancsi OL, Munteanu M, et al. Clinical outcomes of micropulse transscleral cyclophotocoagulation in refractory glaucoma-18 months follow-up. Lasers Med Sci 2020;35(7):1487-1491. DOI: 10.1007/s10103-019-02934-x.

29. Nguyen AT, Maslin J, Noecker RJ. Early results of micropulse transscleral cyclophotocoagulation for the treatment of glaucoma. Eur J Ophthalmol 2020;30(4):700-705. DOI: 10.1177/112067211 9839303.

30. Magacho L, Lima FE, Ávila MP. Double-session micropulse transscleral laser (CYCLO G6) as a primary surgical procedure for glaucoma. J Glaucoma 2020;29(3):205-210. DOI: 10.1097/IJG.00000000 00001426.

31. Magacho L, Lima FE, Ávila MP. Double-session micropulse transscleral laser (CYCLO G6) for the treatment of glaucoma. Lasers Med Sci 2020;35(7):1469-1475. DOI: 10.1007/s10103-019-02922-1.

32. Varikuti VNV, Shah P, Rai O, et al. Outcomes of micropulse transscleral cyclophotocoagulation in eyes with good central vision. J Glaucoma 2019;28(10):901-905. DOI: 10.1097/IJG.0000000000001339.

33. Yelenskiy A, Gillette TB, Arosemena A, et al. Patient outcomes following micropulse transscleral cyclophotocoagulation: intermediate-term results. J Glaucoma 2018;27(10):920-925. DOI: 10.1097/IJG.0000000000001023.

34. Subramaniam K, Price MO, Feng MT, et al. Micropulse transscleral cyclophotocoagulation in keratoplasty eyes. Cornea 2019;38(5):542545. DOI: $10.1097 / \mathrm{ICO} .0000000000001897$.

35. Sarrafpour S, Saleh D, Ayoub S, et al. Micropulse transscleral cyclophotocoagulation: a look at long-term effectiveness and outcomes. Ophthalmol Glaucoma 2019;2(3):167-171. DOI: 10.1016/j. ogla.2019.02.002.

36. Al Habash A, AlAhmadi AS. Outcome of MicroPulse $\left({ }^{\oplus}\right)$ transscleral photocoagulation in different types of glaucoma. Clin Ophthalmol Auckl NZ 2019;13:2353-2360. DOI: 10.2147/OPTH.S226554.

37. Keilani C, Benhatchi N, Bensmail D, et al. Comparative effectiveness and tolerance of subliminal subthreshold transscleral cyclophotocoagulation with a duty factor of $25 \%$ versus $31.3 \%$ for advanced glaucoma. J Glaucoma 2020;29(2):97-103. DOI: 10.1097/ IJG.0000000000001409.

38. Vig N, Ameen S, Bloom $\mathrm{P}$, et al. Micropulse transscleral cyclophotocoagulation: initial results using a reduced energy protocol in refractory glaucoma. Graefes Arch Clin Exp Ophthalmol Albrecht Von Graefes Arch Klin Exp Ophthalmol 2020;258(5):10731079. DOI: 10.1007/s00417-020-04611-0.

39. Sahu DK, Namperumalsamy P, Hilton GF, et al. Bullous variant of idiopathic central serous chorioretinopathy. $\mathrm{Br} J$ Ophthalmol 2000;84(5):485-492. DOI: 10.1136/bjo.84.5.485.

40. Sanchez FG, Lerner F, Sampaolesi J, et al. Efficacy and safety of Micropulse ${ }^{\circledR}$ transscleral cyclophotocoagulation in glaucoma. Arch Soc Espanola Oftalmol 2018;93(12):573-579. DOI: 10.1016/j. oftal.2018.08.003.

41. Dhanireddy S, Yin HY, Dosakayala N, et al. Severe inflammation and hyphema after micropulse diode transscleral cyclophotocoagulation. J Glaucoma 2020;29(6):e50-e52. DOI: 10.1097/IJG.00000000 00001508.

42. Perez $\mathrm{Cl}$, Han $\mathrm{Y}$, Rose-Nussbaumer J, et al. Neurotrophic keratitis after micropulse transscleral diode laser cyclophotocoagulation. Am J Ophthalmol Case Rep 2019;15:100469. DOI: 10.1016/j. ajoc.2019.100469. 
43. Kim J, Sung MS, Park SW. Neurotrophic keratopathy after micropulse transscleral cycloophotocoagulation in a glaucoma patient. Korean J Ophthalmol 2020;35(1):97-98. DOI: 10.3341/kjo.2020. 0103.

44. Prager AJ, Anchala AR. Suprachoroidal hemorrhage after micropulse cyclophotocoagulation diode therapy. Am J Ophthalmol Case Rep 2020;18:100659. DOI: 10.1016/j.ajoc.2020. 100659.
45. Kiyama Y, Nakashima K-I, Inoue T. A case of primary open-angle glaucoma with conjunctival laceration after micropulse transscleral cyclophotocoagulation. J Glaucoma 2020;29(12):e135-e137. DOI: 10.1097/IJG.0000000000001658.

46. Chan PP, Lam MCW, Baig N. Case report - acute corneal subepithelial hydrops (ACSH) during micropulse transscleral cyclophotocoagulation (MPTSC). BMC Ophthalmol 2020;20(1):409. DOI: 10.1186/s12886-020 01669-6. 